\title{
TRICHOBEZOAR IN YOUNG NEPALESE GIRL WITH RAPUNZEL SYNDROME: A CASE REPORT
}

\author{
SHRESTHA R ${ }^{1}$, NEPAL B ${ }^{2}$, PURBEY B ${ }^{3}, \mathrm{KHADKA} \mathrm{D}^{4}$,SAH MK ${ }^{5}$,BARAL ${ }^{5}$, THAPA J ${ }^{5}, \mathrm{SHRESTHA} \mathrm{B}^{3}$,BARAL \\ $\mathrm{RK}^{5}$, POUDEL BN ${ }^{1}$
}

\begin{abstract}
:
Trichobezoar is a gastric foreign body that is composed of the patient's own hair in the stomach. Rapunzel syndrome is an unusual form of trichobezoar in stomach, which extends into any part of the small intestine. We are reporting the case of gastric trichobezoar with thin tail extending into the duodenum in a twenty year oldgirl. She had a history of trichophagia. She presented with epigastric pain and vomiting. Endoscopic removal was attempted but this was unsuccessful. Subsequently the patient underwent exploratory laparotomy and gastrotomy. She recovered well and was discharged with advice for psychiatric consultation.
\end{abstract}

Key word: Trichobezoar, Rapunzel syndrome

Received: 13 October, 2020

Accepted: 25 December, 2020

DOI: https://doi.org/10.3329/bjm.v32i1.51099

\section{Introduction:}

A bezoar is an indigestible mass of foreign material found within the digestive tract, most commonly in the stomach.Bezoars are named based on their components ${ }^{1,2,3}$. Trichobezoar is a collection of hair in the stomach, secondary to impulsive pulling and intake of hair. Small trichobezoars can be removed endoscopically whereas larger one require surgical removal 4,5,6. Rapunzel syndrome is the accumulation of hair in stomach which extends into any part of the small intestine ${ }^{1,2,3}$. Here we present case of a young girl with large trichobezoar that had to be removed surgically.

\section{Case report:}

A 20 year old girl was brought to our department with complaints of abdominal pain, nausea and vomiting, 3-4 episodes per day for the last three months. She recently developed constipation. She used to take her own hair frequently for the last six months during stressful situations.

Her physical examination revealed stable vitals. She was thin built with pallor on examination and she was afebrile. Her hair was short and broken. There was mild tenderness in epigastric region. Cardiorespiratory examination was unremarkable.

Haemoglobin was $10.4 \mathrm{gm} / \mathrm{dl}$ with normal leucocyte count.Peripheral blood film showed mild anisopoikilocytosis, normocytic, normochromic to microcytic hypochromicwith a fewelliptocytes morphology. Total iron was $6.3 \mathrm{ug} / \mathrm{dl}$ ( $\mathrm{N}: 50-170)$ and vitamin B 12(ECI) $182 \mathrm{pg} / \mathrm{ml}$ (normal 239931).Ultrasound of abdomen showed cystic lesion noted in the right adnexa, measures $4.7 \times 4.2 \times 2.5$ cm.(25.5 ml.). Chest x-ray was normal.

She underwent upper gastrointestinal endoscopy for further evaluation.Therstomachwaswas full of hair (Fig.1). Various accessories like Dormia Basket,Crocodile forceps, snare were used to remove hair. Even Argon plasma coagulation probe (linear) was used to fragment hair (Fig.2,3).A small amount of fragment of hair could be removed (Fig.4).

After endoscopic retrieval was unsuccessful, surgical intervention was done with laparotomy. Intraoperatively a firm mass was felt inside the stomach.Approximately ten centimeters of anterior gastrotomy was done, and a 14 X $7 \mathrm{~cm}$.of firm consolidated mass of hair was found inside the

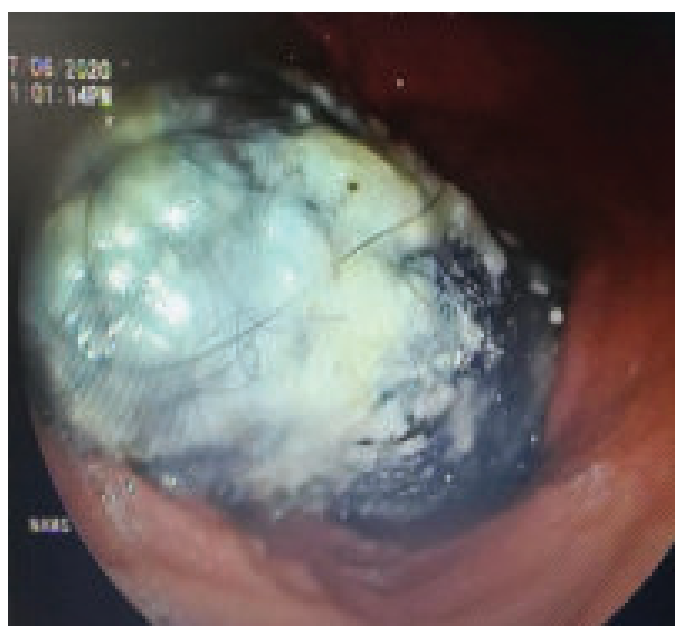

Fig.-1 : Endoscopic view of a large trichobezoar within the stomach. 


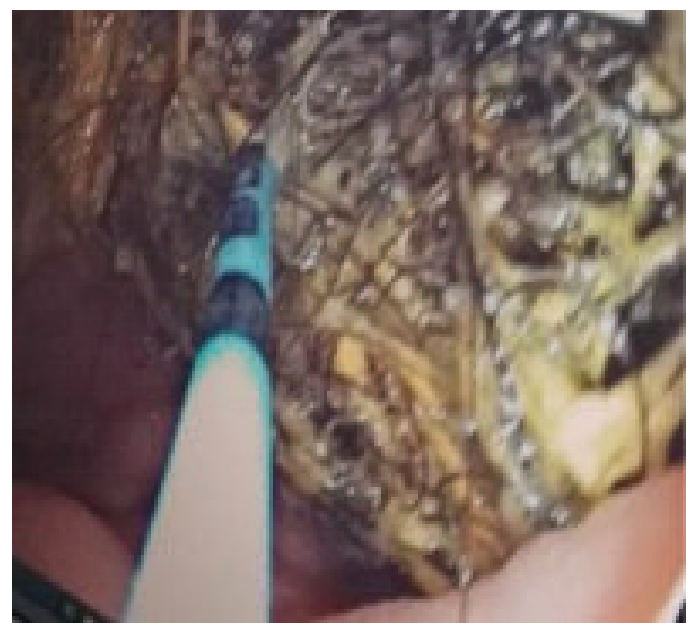

Fig.-2 : Fragmentation wih Argon plasma coagulation (linear probe)

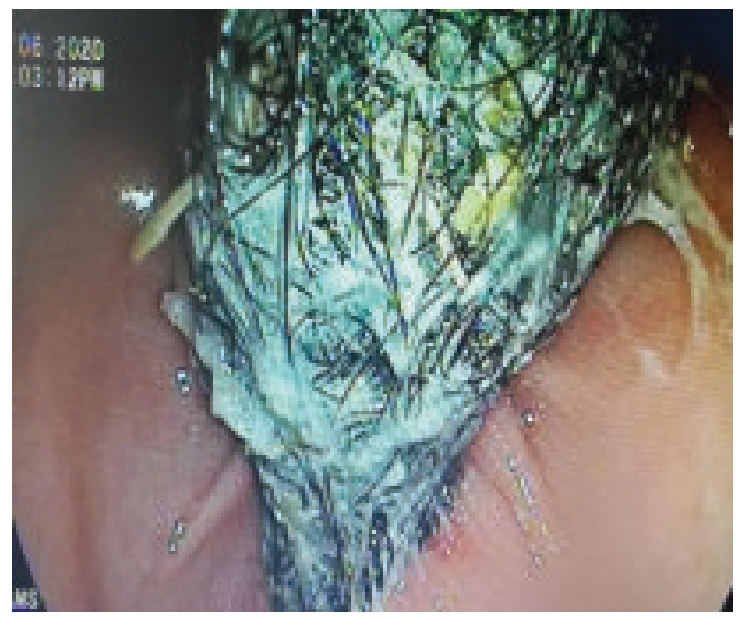

Fig.-3 : Trichobezoar within the stomach.

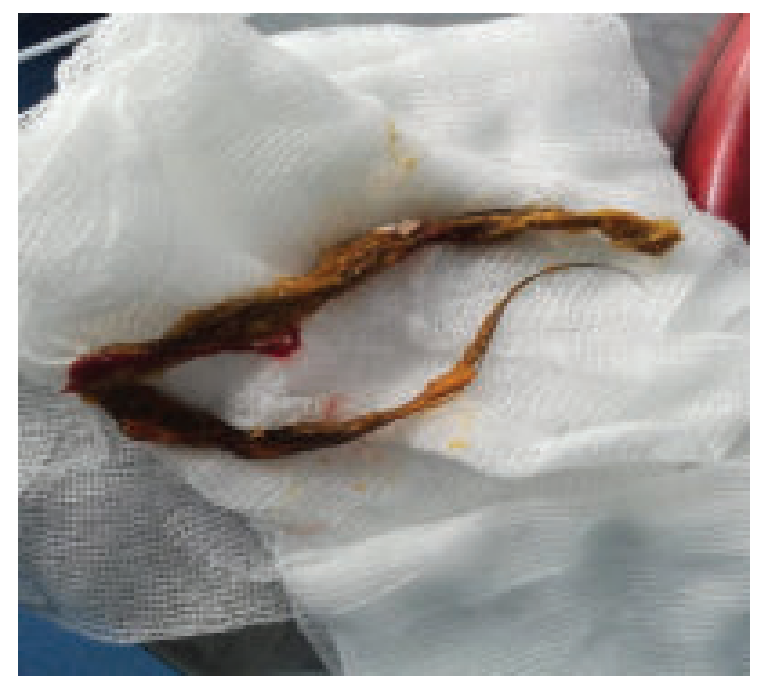

Fig.-4 : Endoscopic removal of fragments of trichobezoar. stomach extending upto first and second part of duodenum(Fig.5 ). The specimen was delivered intact(Fig.5), no other hairy mass was felt upto the second part of duodenum. Patient was allowed oral intake on the third day.Five days later she was discharged.Then patient was referred to psychiatry hospital for further evaluation and management to prevent the recurrence.

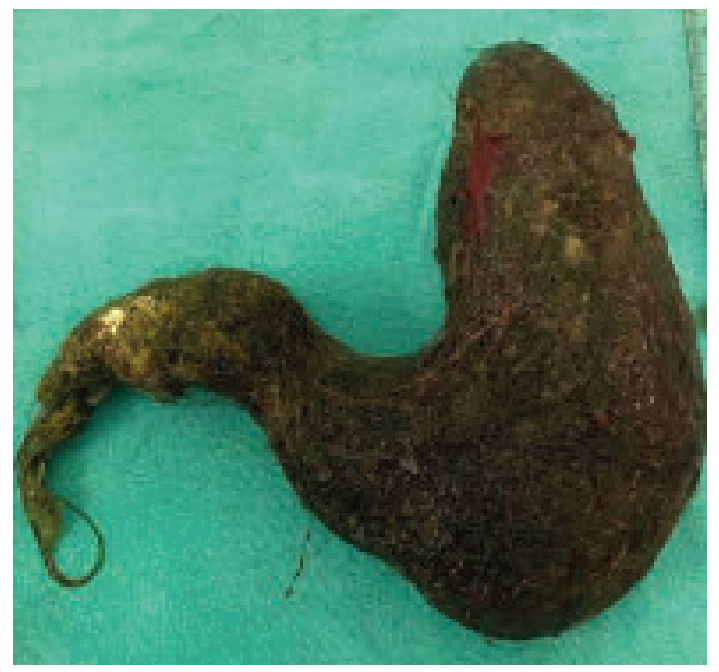

Fig.-5 : Image of operative specimen of large trichobezoar.

\section{Discussion:}

A bezoar is a conglomeration of food or foreign material which accumulate and remain within the gastrointestinal tract.The word "bezoar" comes from the Arabic word "bedzehr" or the Persian word "Padzhar"meaning "protecting against a poison".In history bezoars from animal guts were used as precious stones, antidotes to poisons.It is the part of traditional Chinese medicine ${ }^{3,7,8}$. They have been classified into four groups: phytobezoars(caused by vegetables), trichobezoars (caused by hair), lactobezoars(caused by milk), miscellaneous (caused by medication; pharmacobezoars, tissue papers, sand or fungus). The first reference to a bezoar in a human was in 1779 during an autopsy of a patient who died from gastric perforation and peritonitis ${ }^{3,8}$.

Rapunzel was the name of the maiden in the "Grimm brothers"fairy tale in 1812, whose long hair flowed out of her prison tower allowing her prince to rescue her 9 . Rapunzel syndrome was first described in literature by Vaughan et al.in $1968^{8}$. Fewer than a hundred cases of Rapunzel syndrome have been reported in the literature since the initial description. In Rapunzel syndrome, the trichobezoar extends through the 
pylorus into the small intestine ${ }^{8}$. Most cases of trichobezoar are reported in females with traditional long hair,one reported male case who ate the hair of his sister ${ }^{11}$.Most patients with trichobezoar suffer from psychiatric disorder including trichotillomania (pulling out their own hair)and trichophagia (eating hair).

The formation of trichobezoar in these patients depends upon the quantity and duration of trichophagia. On an average only $1 \%$ patients with trichophagia develop trichobezoar 6,12,13. As more and more hair accumulates, a ball of hair is formed which becomes too large to escape and results in gastric atony and subsequently takes the shape of stomach ${ }^{14}$. The acid contents of the stomach denature hair protein and give the bezoar a jet black color ${ }^{15}$. The most common presenting signs are abdominal pain, nausea, vomiting, obstruction, peritonitis and less commonly patients have presented with weight loss, anorexia, hematemesis and intussusception. Complications include gastric ulceration, obstructive jaundice, acute pancreatitis and gastric emphysema 6,15.

Diagnostic modalities include ultrasound, computed tomography(CT). CT scan has high accuracy rate but ultrasound in such cases is very sensitive ${ }^{20}$. Upper gastrointestinal endoscopy remains the examination of choice in the diagnosis of intragastric trichobezoar as it allows visualizing the hair threads and trying an extraction ${ }^{21}$.Various therapeutic modalities have been proposed to treat trichobezoar.

Gorteret al.in a retrospective review of 108 cases of trichobezoar evaluated the variable treatments tried in these cases.It was noted that $5 \%$ of attempted endoscopic removal were successful ( small trichobezoar may respond to endoscopic fragmentation and vigorous lavage), 75\% of attempted laparoscopic surgical extractionwere successful. Laparotomy followed by gastrotomy was $99 \%$ successful 22,23.

Specialized bezotomes (device that pulverize bezoars ) and bezotriptors ( devices that fragment bezoars by acoustic waves ) have been used to fragment large and solid trichobezoars ${ }^{21,23}$. Various other methods like extracorporal shock wave lithotripsy, intra- gastric administration of enzymes ( pancreatic lipase,cellulose) and medications metoclopramide, acetylcysteine, demonstrate have been used with varying success 24 .

\section{Conclusion:}

Rapunzel syndrome should be considered as differential diagnosis in young girl presenting with abdominalpain,vomiting and anaemia.Early diagnosis can prevent these complications. Psychiatric evaluation, counselling and treatment are helpful in preventing recurrence. Trichobezoar often coexist withpsychiatric illness.

\section{References:}

1. Williams RS. The fascinating history of bezoars.Mwd J Aust 1986:145:613-4. https://doi.org/10.5694/ j.1326-5377.1986.tb139511.x PMid:3540541

2. Wang C, ZhaoX, MaoS, Wang Y,Cui X. Management of SAH with traditional Chinese medicine in China.Neurol Res 2006;28:436-444[PubMed][Google Scholar]. https:/ / doi.org/10.1179/016164106X115044 PMid:16759447

3. Naik S, Gupta V, NaikS , Rangple A,Chaudhary AK, JainP, Sharma AK. Rapunzel syndrome reviewed and redefined.Dig Surg 2007;24:157-161. https://doi.org/ 10.1159/000102098 PMid:17476105

4. Saeed ZA, Ramirez FC, Hepps KS et al. A method for the endoscopic retrieval of trichobezoars. Gastrointest Endosc, 1993;39:698-700 https://doi.org/10.1016/ S0016-5107(93)70226-6

5. Dietrich NA,Gau FC (1985) Postgastrectomy phytobezoar- endoscopic diagnosis and treatment. ArchSurg 120:432-435 https://doi.org/10.1001/ archsurg.1985.01390280026006 PMid:3985787

6. Phillips MR, Zaheer S, Grugas Gt. Gastrictrichobezoar: Case report and literature review.Mayo Clinic Proc 1998;73:653-6. https://doi.org/10.4065/73.7.653 https://doi.org/10.1016/S0025-6196(11)64889-1

7. DeBakeyM,OchsnerA.Bezoars and Concretions: A comprehensive review of literature with analysis of 303 collected cases and presentation of 8 additional cases.Surgery 1938;4:934-63.

8. Vaughn ED Jr., Sawyers JL, Scott HW Jr.The Rapunzel syndrome-an unusual complication of intestinal bezoar.Surgery 1968;63:339-43.

9. Grimm Brothers:Rapunzel.Translated by GodwinJonesR.Richmond,Virginia Commonwealth University Department of Foreign Languages, 1994-1999.

10. Shorter E.A historical dictionary of Psychiatry.New York: Oxford University Press;2005.

11. Hirugade ST, Talpallikar MC, Deshpande AV, Gavali JS, Borwankar SS: Rapunzel syndrome with a long tail. Indian J Pediatr 2001:68:895-896. https://doi.org/ 10.1007/BF02762123 PMid:11669043

12. Irving PM, Kadirkamanathan SS, Priston AV, Blanshard C. Education and imaging. Gastrointestinal :Rapunzel syndroe. J Gastroenterol Hepatol.2007;22:2361. https://doi.org/10.1111/j.1440-1746.2007.05230.x PMid: 18031403

13. Gonuguntla V, Joshi DD. RapunzelSyndrome.A comprehensive review of an unusual case of Trichobezoar. Clin Med REs 2009;7:99-102.https:// doi.org/10.3121/cmr.2009.822 PMid:19625498 PMCid: PMC2757434 
14. Christenson GA,Mackenzie TB, Mitchell JE.Characteristics of 60 adult hair pullers.Am J Psychiatry 1991;148:365-70. https://doi.org/10.1176/ ajp.148.3.365 PMid:1992841

15. Sharma V,Sahi RP,Misra NC.Gastro-intestinal bezoar.J Indian Med Assoc 1991;89:338-9.

16. Jiledar Singh G,Mitra SSK. Gastric perforation secondary to recurrent trichobezoar.Indian J Pediatr 1996;63:689-691. https: / / doi.org/10.1007/BF027 30823 PMid:10830041

17. KlipfelAA,KesslerE,ScheinM.Rapunzel syndrome causing gastric emphysema and small bowel obstruction.Surgery 2003;133:120-121. https:// doi.org/10.1067/msy.2003.41 PMid:12563250

18. SidhuBS,SinghG, KhannaS.Trichobezoar.J Indian Med Assoc 1993;91:100-1.

19. DebakeyM,OschnerA.Bezoars and concretions: Comprehensive review of literature with analysis of 303 collected cases and presentations of 8 additional cases.Surgery. 1939;5:132-60.
20. Sharma UK, Sharma Y, Chhetri RK, Makaju RK, Chapagain S, ShresthaR. Epigastric mass in a young girl: Trichobezoar:Imaging Diagnosis. Nepal Med Coll J 2006: 8:211-2.

20. Wang YG,SeitzU,LiZLSoehendraN,QiaoXA.Endoscopic management of huge bezoars. Endoscopy 1998:30:371374. https://doi.org/10.1055/s-2007-1001285 PMid:9689511

22. A.Gossum,M.Delhaye,M.Cremer.Failure of nonsurgical procedures to treat gastric trichobezoar. Endoscopy, 21(1989),p.11. https://doi.org/10.1055/s-20071012916 PMid:2565222

23. SaeedZA,RamirezFC,Hepps KS et al (1993).A method for the endoscopic retrieval of trichobezoars. Gastrointest Endosc 39:698-700. https://doi.org/ 10.1016/S0016-5107(93)70226-6

24. Groenewald CB,Smoot RL,Farley DR.A football-sized gastric mass in a healthy teen.CotempSurg 2006:62: $531-534$. 